\title{
Evaluación de la fitotoxicidad de efluentes industriales
}

\author{
María Elena Sáenz ${ }^{1}$, María del Carmen Tortorelli ${ }^{1}$ y Lauce Ruben Freyre ${ }^{2}$
}

${ }^{1}$ Programa de Investigación en Ecotoxicología, Departamento de Ciencias Básicas, Universidad Nacional de Luján. Casilla de Correo 221 (6700) Luján (B) Argentina. ${ }^{2}$ Instituto de Limnología (ILPLA) "Dr. Raúl A. Ringuelet" Facultad de Ciencias Naturales y Museo Universidad Nacional de La Plata, Av. Calchaqui Km 23500 Florencio Varela, Apartado Postal 712 (1900) La Plata.

\section{RESUMEN}

Se realizó la evaluación de la fitotoxicidad de efluentes industriales que representan las principales actividades industriales llevadas a cabo en la zona de influencia del río Luján. (Pcia. de Buenos Aires, Argentina) sobre las especies de algas Clorofitas Scenedesmus quadricauda y Raphidocelis subcapitata. El efluente proveniente de la industria de producción de plásticos y herbicidas no resultó tóxico para ninguna de las dos especies estudiadas, por el contrario, las poblaciones algales expuestas a concentraciones crecientes del efluente presentaron una estimulación del crecimiento respecto a las poblaciones controles. Este efluente podría ocasionar, problemas de eutrofización en el ambiente acuático receptor. Los efluentes provenientes de la industria productora de colorantes y de la industria de curtido de cueros resultaron tóxicos para las especies evaluadas. El efluente que presentó la mayor toxicidad fue el correspondiente a la industria de curtidos de cueros, se realizaron estimaciones de la carga tóxica aportada por este efluente al río Luján, sistema acuático receptor del mismo. Las evaluaciones ecotoxicológicas realizadas con muestras líquidas provenientes del río Luján, permiten concluir que existiría una reducción de la toxicidad in situ por efectos de dilución, reduciendo el impacto de este efluente sobre el sistema acuático receptor. Respecto a la sensibilidad de las especies utilizadas, la cepa de Scenedesmus quadricauda aislada de las nacientes del río Luján, resultó ser la más sensible, tanto para los índices de toxicidad aguda como crónica.

Palabras clave: Toxicidad, efluentes industriales, algas, agua dulce, impacto ambiental.

\begin{abstract}
Phytotoxicity assessments were performed of the main industrial effluents discharging into the Luján river valley (province of Buenos Aires, Argentina) using the green algae species Scenedesmus quadricauda and Raphidocelis subcapitata as indicator species. The effluent from the plastic and herbicide industry was not toxic to both algal species. On the contrary, algal cultures exposed to effluents showed stimulation of algal growth compared to control cultures. This effluent, however, could cause eutrophication in the receiving system. Effluents from the coloring and tannery industries were toxic to both species. Tannery effluentt was the most toxic. The ecotoxicological assessment of river water samples showed that a reduction of toxicity due to dilution could occur, decreasing the impact of the tannery effluent on the freshwater receiving system. The strain of Scenedesmus quadricauda isolated from samples at the source of Luján river was the most sensitive to both acute and chronic toxicity.
\end{abstract}

Keywords: Toxicity, industrial effluent, algae, freshwater, environmental impact

\section{INTRODUCCIÓN}

El crecimiento de la población humana ha generado un aumento en la producción industrial a nivel mundial, determinando el incremento de las descargas de aguas residuales tanto industriales como cloacales. Estos efluentes contienen una gran variedad de sustancias orgánicas e inor- gánicas, las que potencialmente pueden producir efectos tóxicos sobre los organismos acuáticos.

Las aguas residuales constituyen una mezcla compleja de sustancias orgánicas e inorgánicas. Los efectos que producen sobre los organismos acuáticos resultan de una combinación e interacción entre sus constituyentes no existiendo, en general, una sustancia única que tenga un efecto 
dominante. La evaluación de la toxicidad del efluente completo refleja la interacción de distintas variables (Dorn \& Compernolle, 1995; Grothe et al., 1996).

Algunas sustancias pueden ser muy tóxicas en forma aislada pero su efecto tóxico puede estar mitigado o aumentado dando origen a acciones antagónicas y/o sinérgicas, cuando se encuentra formando parte de un efluente debido a la interacción con otros compuestos (Ferrari et al., in press; Kallqvist \& Svenson, 2003; Juneau et al., 2003)

Algunos parámetros físicos de las aguas residuales como el $\mathrm{pH}$ y la temperatura pueden afectar los organismos acuáticos como fue demostrado por Joubert (1980) y Eloranta \& Kettunen (1979).

La evaluación toxicológica de las aguas residuales mediante organismos acuáticos ha demostrado ser de utilidad en la cuantificación de los efectos tóxicos producidos por estas mezclas complejas de contaminantes. Estas evaluaciones permiten detectar efectos que no son estimados a través de la caracterización físicoquímica de los efluentes.

Las algas constituyen organismos importantes para su introducción en la batería de organismos utilizada para la determinación de la calidad de las aguas, ya que las aguas residuales frecuentemente presentan exceso de nutrientes y otros tóxicos los cuales generan efectos que las evaluaciones con microinvertebrados y peces no detectarían (Walsh \& Banher, 1980). Los efectos de estimulación de crecimiento observados en algas son fundamentales para la evaluación de los efectos negativos de los efluentes con alto contenido en nutrientes, responsables de potenciales problemas de eutrofización. Existen antecedentes bibliográficos enfatizando el importante papel de las algas en la determinación de la fitotoxicidad de los efluentes industriales (Claesson, 1984; Thomas et al., 1986; Amblard et al., 1990; Lewis, 1992; Arauzo et al., 2000; Tarlan et al., 2002; Sponza, 2003; Bruno \& Eklund, in press)

Las algas han resultado ser más sensibles que los microinvertebrados y peces en el caso de mezclas complejas como los efluentes industriales y cloacales (Ekholm \& Krogerus, 1998; Burridge \& Bidwell, 2002). Las diferentes respuestas encontradas incluyen efectos inhibitorios, de estimulación y cambios en el tamaño y en la morfología celular, como células alargadas, cloroplastos anormales y evidencias de mitosis sin citocinesis. Los efluentes con contenidos en Cadmio han producido cambios ultraestructurales a nivel de las mitocondrias en varias especies de Clorofitas (Walsh \& Merrill, 1984). Estudios realizados acerca de los efectos de las descargas de aguas residuales sobre la comunidad fitoplanctónica, han demostrado que puede existir modificaciones en los procesos metabólicos algales irreversibles sin que resulten afectadas la composición y la estructura de la comunidad (Couture et al., 1987). Estudios realizados a lo largo de un gradiente de contaminación en el río Luján, se observó que el efecto de los mismo fue alterar la distribución y proporciones relativas de las algas, más que la composición específica de base (del Giorgio, 1991).

Respecto de la problemática del vertido de aguas residuales en la zona de la cuenca del río Luján, este sistema acuático resulta el receptor de los efluentes residuales de la mayoría de los establecimientos industriales ubicados en el Partido de Pilar y el Partido de Luján (Provincia de Buenos Aires). Según un relevamiento realizado por De Pietri (1999) gran parte de las aguas residuales de los 106 establecimientos que se encuentran en el Parque Industrial Pilar (Partido de Pilar - Provincia de Buenos Aires) son vertidos en el tramo inferior del río Luján. Del total de establecimientos, las industrias petroquímicas, químicas, farmacéuticas, productoras de pigmentos textiles, plaguicidas y herbicidas representan el $26 \%$; las industrias alimenticias, el $18 \%$ y textiles y curtiembres, el $4 \%$. La mayoría de los establecimientos industriales que se encuentran en el Partido de Luján, vierten sus aguas residuales directamente o indirectamente (a través de arroyos afluentes) en el tramo medio del río Luján. Según la información provista por la Municipalidad de Luján, los 
establecimientos metalúrgicos y cromados representan un $28 \%$; las industrias alimenticias, un $25 \%$; las textiles, el $25 \%$; elaboración de plásticos un, $9 \%$; químicas, un $6 \%$ y curtiembres, el 3 \% (Sáenz, 1992). Lamentablemente, esta última fuente de información no cuenta con datos actualizados acerca de los caudales de cada una de las categorías señaladas y en algunos casos los desconoce; por lo cual no es posible conocer la importancia cuantitativa relativa o absoluta. Sin duda, esta información resultaría valiosa y permitiría conocer la exacta influencia de los vertidos de cada actividad sobre el tramo medio del río Luján.

En el presente trabajo se realizaron evaluaciones de la toxicidad de aguas residuales que representan las principales actividades industriales llevadas a cabo en la zona de influencia del río Luján. Las aguas residuales evaluadas correspondieron a una industria de producción de plásticos y herbicidas, industria de producción de colorantes e industria del curtido de cueros (curtiembre).

\section{MATERIAL Y MÉTODOS}

Se realizaron ensayos de 96 horas de duración, en condiciones controladas de laboratorio exponiendo cultivos algales uniespecíficos de las especies Scenedesmus quadricauda y Raphidocelis subcapitata a diferentes concentraciones de los efluentes evaluados según los procedimientos descriptos en US EPA (1989) y US EPA (1996). La cepa de Scenedesmus quadricauda (División Chlorophyta, Orden Chlorococcales) fue aislada a partir de muestras recolectadas en las nacientes del río Luján. Se aplicaron técnicas de aislamento y purificación (Sáenz, 2000). La verificación taxonómica de la misma fue realizada en la Cátedra de Botánica Sistemática I de la Facultad de Ciencias Naturales y Museo UNLP. La cepa utilizada de Raphidocelis subcapitata (División Chlorophyta, Orden Chlorococcales) provino del Cepario de la Companhia de Saneamento Ambiental del Estado de São Paulo (CETESB), Brasil. Ambas especies se mantienen actualmente en el Cepario del
Programa de Investigaciones en Ecotoxicología de la Universidad Nacional de Luján.

Los efluentes utilizados en las evaluaciones fueron recolectados luego de la salida de los sistemas de tratamientos de las aguas residuales, previa a su cloración. Los mismos fueron transportados refrigerados al laboratorio, iniciando las evaluaciones de toxicidad dentro de las 24 horas desde su llegada. Previo a su utilización, los efluentes se filtraron a través de un filtro Whatman GF/C. Posteriormente, se agregaron al efluente puro las soluciones de macronutrientes y micronutrientes que constituyen el medio nutritivo algal utilizado como control, en las mismas proporciones. Este mismo medio fue el agua de dilución utilizada para la preparación de las diferentes soluciones de prueba de los efluentes evaluados. Según lo indicado con anterioridad, estos son los procedimientos descriptos en US EPA (1989) y US EPA (1996) para las evaluaciones de toxicidad algal de efluentes industriales. El objetivo de la adición de los nutrientes al efluente analizado es descartar las respuestas inhibitorias del crecimiento debido a la falta de nutrientes en el efluente. De esta manera tanto los controles como las distintas diluciones de los efluentes presentan por lo menos las mismas concentraciones de nutrientes.

Las muestras provenientes del río Luján fueron pasadas a través de una red de $25 \mu$ de tamaño de malla con el objetivo de remover los organismos presentes en las mismas. No se realizó la filtración de las muestras para no retirar los sólidos en suspensión y de esta manera contar con una muestra representativa, ya que éstos pueden influenciar notablemente la toxicidad de los efluentes. Los parámetros físicos químicos de los efluentes utilizados y de las muestras del río Luján se determinaron según APHA (1992).

La exposición de los cultivos algales se realizó a $22{ }^{\circ} \mathrm{C} \pm 1{ }^{\circ} \mathrm{C}$ de temperatura, $3000 \mathrm{lux} / \mathrm{cm}^{2}$ de intensidad lumínica y con agitación de 100 r.p.m. en una cámara provista con agitador orbital marca Forma. Se determinó la densidad celular cada 24 horas. Las curvas de crecimiento fueron ajustadas al modelo de regresión exponencial según la ecuación: $N_{t}=N_{0} \mathrm{e}^{r t}$ donde 


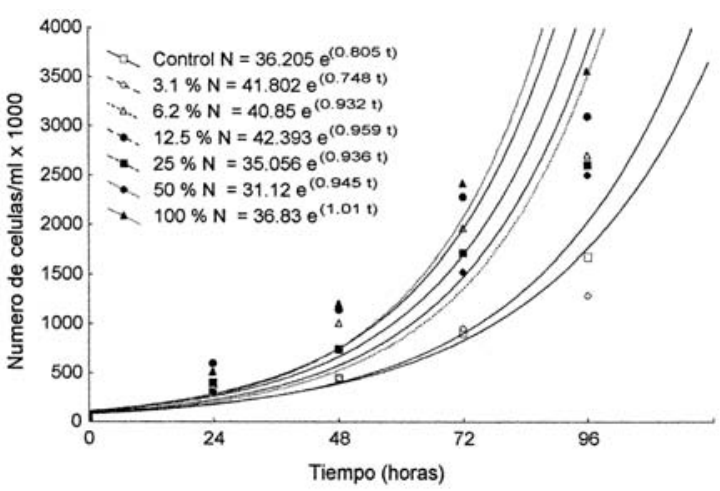

Figura 1. Curvas de crecimiento de poblaciones de Scenedesmus quadricauda expuestas a un efluente de una industria de producción de plásticos y herbicidas. Growth curves of Scenedesmus quadricauda cultures exposed to effluent from a production plant of plastics and herbicides.

$r$, tasa de crecimiento exponencial, resulta la pendiente del modelo. El coeficiente de determinación $\left(r^{2}\right)$ obtenido en cada caso fue utilizado como criterio para evaluar el ajuste del modelo a los datos de crecimiento.

Los resultados obtenidos fueron analizados mediante análisis de la varianza (ANOVA) de un factor, combinado con test de Dunnet y de Tuckey mediante el programa TOXSTAT Version 3.5 (West Inc \& Gulley, 1996). Los valores de las $\mathrm{EC}_{50}$ (concentración efectiva), fueron estimados mediante análisis probit. Para la estimación del LOEC (concentración efectiva más baja) y NOEC (concentración no efectiva) fue utilizado el test de Dunnet (EPA, 1989).

\section{RESULTADOS}

\section{Industria de producción de plásticos y herbicidas}

Los parámetros físico-químicos del efluente utilizado en los ensayos de toxicidad se indican en la Tabla 1. Este efluente es el efluente final posterior al tratamiento biológico por un sistema de lodos activados y anterior al proceso de cloración.

El efluente proveniente de una industria de producción de plásticos y herbicidas no resultó
Tabla 1. Parámetros físico-químicos de los efluentes utilizados en las evaluaciones de fitotoxicidad. $\mathrm{PH}$ : plásticos y formulación de herbicidas; C: colorantes; CUR: curtido de cueros. Physical-chemical parameters of the effluents used in phytotoxicity tests. $\mathrm{PH}$ : plastics and herbicides industry effluent; C: Coloring plant effluent; CUR: tannery industry effluent.

\begin{tabular}{lccc}
\hline PARÁMETRO & PH & C & CUR \\
\hline Salinidad $(\%)$ & 1 & 6 & 6 \\
Conductividad $(\mu \mathrm{S} / \mathrm{cm})$ & 1140 & 9000 & 10500 \\
Dureza $\left(\mathrm{mg} \mathrm{CaCO}_{3} / \mathrm{L}\right)$ & 143.62 & 600.75 & 120 \\
pH & 7.60 & 7.93 & 8.03 \\
Oxigeno disuelto $(\mathrm{mg} / \mathrm{l})$ & 9.19 & 3.50 & 5 \\
DQO (mg/l) & 104 & 584 & 200 \\
DBO $(\mathrm{mg} / \mathrm{l})$ & 3.39 & 60 & 42 \\
Nitratos $(\mathrm{mg} / \mathrm{l})$ & 14.4 & 28.78 & 4 \\
Fosfatos $(\mathrm{mg} / \mathrm{l})$ & 11.11 & 13.8 & 0.4 \\
Cromo total $(\mathrm{mg} / \mathrm{l})$ & - & - & 0.9 \\
\hline
\end{tabular}

tóxico para ninguna de las dos especies estudiadas (Figs. 1 y 2). Las poblaciones algales expuestas a concentraciones crecientes del efluente presentaron una estimulación del crecimiento respecto a las poblaciones controles. Como se puede observar en las tasas de crecimiento de las respectivas poblaciones algales, el incremento de este parámetro es evidente y resulta ser significativamente diferente de las poblaciones controles (ANOVA - Test de Dunnett, $\mathrm{p}<0.05$ ).

Las poblaciones de Scenedesmus quadricau$d a$ presentaron una estimulación de crecimiento entre un 38 y $53 \%$ al final del ensayo a concentraciones crecientes del efluente en el orden del $6 \%$ hasta el $100 \%$ (efluente puro). La población expuesta a una concentración de efluente del $3 \%$ del efluente presentó una leve disminución de crecimiento a las 96 horas, que no resultó significativa respecto del crecimiento desarrollado por la población control. Si bien el efluente ejerció un efecto de estimulación, el crecimiento algal tuvo un menor ajuste al modelo exponencial, encontrándose coeficientes de determinación entre 0.84 y 0.86. (Fig. 1).

Las poblaciones de Raphidocelis subcapitata respondieron de forma similar a la indicada para Scenedesmus quadricauda. En este caso las poblaciones expuestas a concentracions entre 3.1 y $12.5 \%$ presentaron una disminución 


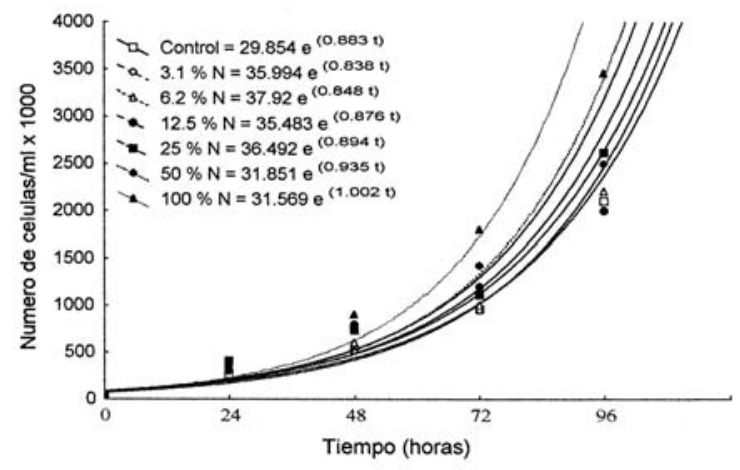

Figura 2. Curvas de crecimiento de poblaciones Raphidocelis subcapitata expuestas a un efluente de industria de producción de plásticos y herbicidas. Growth curves of Raphidocelis subcapitata cultures exposed to effluent from a production plant of plastics and herbicides.

en la tasa de crecimiento entre un 0.007 y $4 \%$ al final del ensayo, que no resultó significativa respecto del control. A las 96 horas, todas las poblaciones presentaron una estimulación significativa del crecimiento respecto al control, en presencia de concentraciones iguales y superiores a $25 \%$ de efluente. El efluente puro causó una estimulación del crecimiento, presentando una tasa de crecimiento $13 \%$ superior al control. En este caso el crecimiento poblacional presentó un mejor ajuste al modelo exponencial con coeficientes de determinación entre 0.90 y 0.93 (Fig. 2).

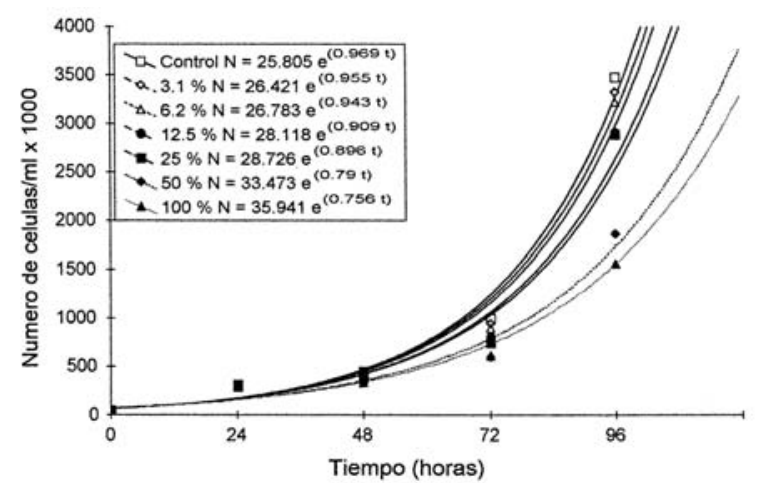

Figura 3. Curvas de crecimiento de poblaciones de Scenedesmus quadricauda expuestas a un efluente de una planta de producción de colorantes textiles. Growth curves of Scenedesmus quadricauda cultures exposed to effluent from a coloring production plant.
Industria de producción de colorantes textiles

Los parámetros físico-químicos del efluente final de esta industria se indican en la Tabla 1.

Las poblaciones de Scenedesmus quadricau$d a$ expuestas a concentraciones mayores de $3.1 \%$ presentaron una disminución del crecimiento algal, significativamente diferente del crecimiento de las poblaciones controles hacia la finalización de la exposición (ANOVA - Test de Dunnett, $\mathrm{p}<0.05$ ) (Fig. 3).

Las poblaciones de Raphidocelis subcapitata expuestas a 3.1 y $6.2 \%$ de efluente no presentaron una disminución significativa del crecimiento algal, respecto del crecimiento de la población control (ANOVA - Test de Dunnett, $\mathrm{p}<0.05$ ). Las poblaciones expuestas a concentraciones superiores a $12.5 \%$ presentaron una disminución significativa del crecimiento en relación con el crecimiento de las poblaciones controles al cabo de 96 horas de exposición (ANOVA - Test de Dunnett, $\mathrm{p}<0.05$ ) (Fig. 4).

\section{Industria de curtido de cueros (curtiembre)}

Los parámetros físico-químicos del efluente final se indican en la Tabla 1. Las poblaciones algales de Scenedesmus quadricauda expuestas a concentraciones del efluente iguales y superiores al $3 \%$ presentaron una inhibición significati-

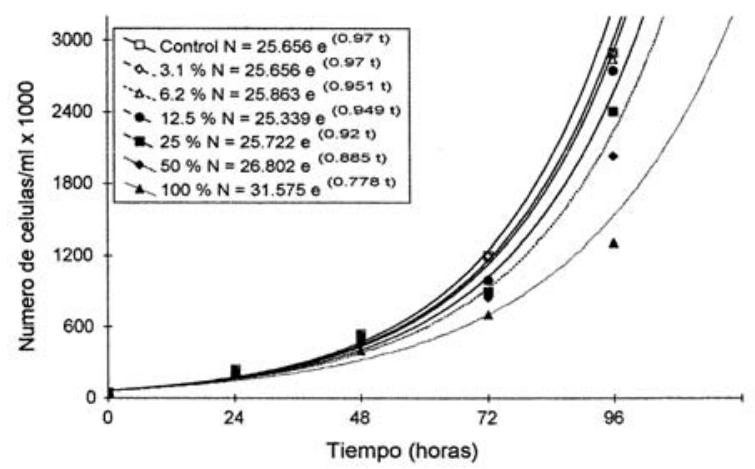

Figura 4. Curvas de crecimiento de poblaciones de Raphidocelis subcapitata expuestas a un efluente de una planta de producción de colorantes textiles. Growth curves of Raphidocelis subcapitata cultures exposed to effluent from a coloring production plant. 


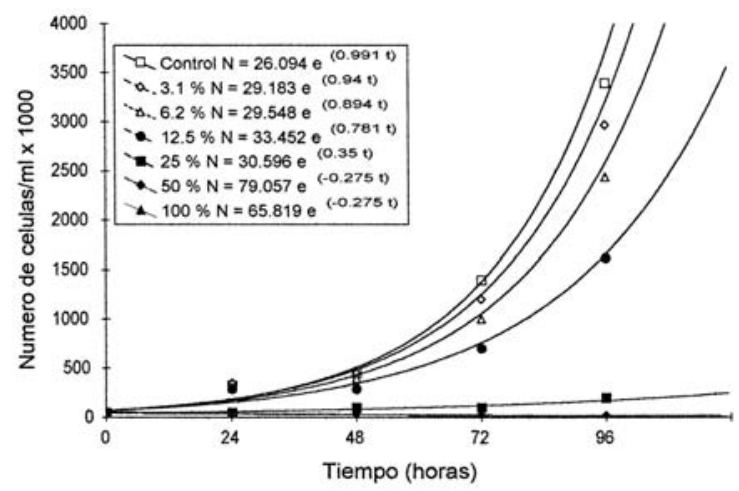

Figura 5. Curvas de crecimiento de poblaciones de Scenedesmus quadricauda expuestas a un efluente de una curtiembre. Growth curves of Scenedesmus quadricauda cultures exposed to effluent from a tannery industry.

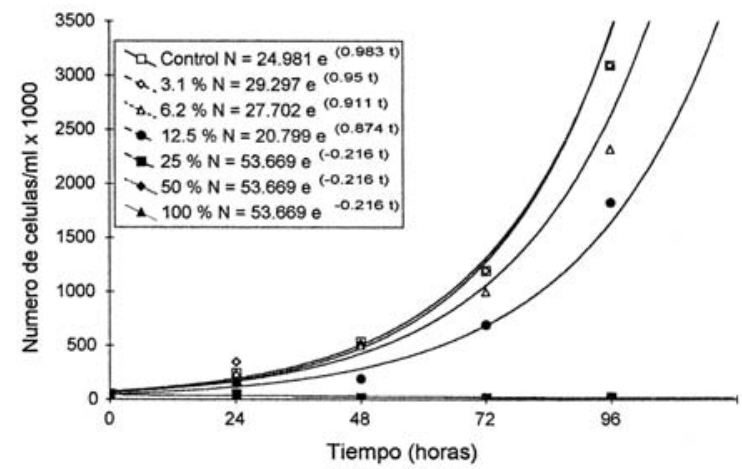

Figura 6. Curvas de crecimiento de poblaciones de Raphidocelis subcapitata expuestas a un efluente de una curtiembre. Growth curves of Raphidocelis subcapitata cultures exposed to effluent from a tannery industry.

va de las tasas de crecimiento respecto de las poblaciones controles (ANOVA - Test de Dunnett, $\mathrm{p}<0.05$ ). Las poblaciones incubadas en presencia de 50 y $100 \%$ del efluente presentaron una inhibición total del crecimiento desde el inicio de la evaluación (Fig. 5). En el caso de Raphidocelis subcapitata las poblaciones algales expuestas a concentraciones iguales y superiores a la menor concentración ensayada $(3.1 \%)$ presentaron una disminución significativa en la tasas de crecimiento respecto a las poblaciones controles (ANOVA - Test de Dunnett, $\mathrm{p}<0.05$ ). Además, no se registró crecimiento algal en las poblaciones expuestas a concentraciones mayores e iguales al $25 \%$ del efluente (Fig. 6).
Los efluentes provenientes de la industria productora de colorantes (C) y de la industria de curtido de cueros (CUR) resultaron tóxicos para las especies evaluadas. Los índices de toxicidad estimados en cada caso se indican en la Tabla 2

\section{DISCUSIÓN}

La toxicidad de los efluentes evaluados puede deberse a la presencia de sustancias tóxicas o por la elevada concentración de sustancias orgánicas e inorgánicas, reflejadas en los valores de conductividad y DQO. Frecuentemente la elevada salinidad de los efluentes es la causante de la toxicidad de los mismos (Dorn \& Compernolle, 1995).

En el caso de la industria de curtido de cueros la toxicidad podría estar vinculada con la presencia de Cromo en el efluente final. Debido a que esta industria posee una planta de tratamiento en óptimas condiciones de funcionamiento, el Cromo total determinado corresponde en su mayor parte a $\mathrm{Cr}^{+3}$. Si bien el $\mathrm{Cr}^{+3}$ presenta menor toxicidad que el $\mathrm{Cr}^{+6}$, los valores de $\mathrm{EC}_{50}$ -96 horas para Raphidocelis subcapitata se ha estimado en $0.32 \mathrm{mg} / \mathrm{l}$ (Greene et al., 1988)

En líneas generales puede decirse que la presencia de sustancias tóxicas en los efluentes se debe al mal funcionamiento de los sistemas de tratamiento, a la incorrecta selección del mismo ó a la necesidad de generar algún tipo de tratamiento químico, adicional al biológico existente, aplicado al efluente a efectos de eliminar la causa de su toxicidad. En este contexto, la aplicación de los ensayos ecotoxicológicos con organismos acuáticos, resulta una herramienta de control para monitorear la eficiencia de un sistema depurativo y saber en que situaciones se está generando o no un efluente seguro para el ambiente.

Debido a que el efluente que presentó la mayor toxicidad fue el correspondiente a la industria de curtidos de cueros, se realizaron estimaciones de la carga tóxica aportada por este efluente al río Luján, sistema acuático receptor del mismo. 
Tabla 2. Indices de toxicidad (\%) al cabo de 96 horas de exposición para ambas especies algales. Industria productora de colorantes (C), Industria de curtido de cueros (CUR). Entre paréntesis se indican los límites de los intervalos de confianza al $95 \%$. Toxicity of effluents endpoints (\%) after $96 \mathrm{~h}$ to both species. Coloring plant $(C)$. Tannery industry (CUR). In parentheses, $95 \%$ confidence intervals.

\begin{tabular}{lcccc}
\hline & $\begin{array}{c}\text { Scenedesmus } \\
\text { quadricauda }\end{array}$ & \multicolumn{2}{c}{$\begin{array}{c}\text { Raphidocelis } \\
\text { subcapitata }\end{array}$} \\
\hline & $\mathrm{C}$ & CUR & $\mathrm{C}$ & CUR \\
\hline $\mathrm{EC}_{50}-96 \mathrm{hrs}$ & 79 & 9.4 & 88 & 11.3 \\
NOEC & $(60-98)$ & $(8-11)$ & $(71-114)$ & $(9.8-13)$ \\
LOEC & 1.5 & 1.5 & 6.2 & 3.1 \\
& 3.1 & 3.1 & 12.5 & 6.2 \\
\hline
\end{tabular}

La estimación de la carga tóxica permite evaluar la contribución de cada efluente a los sistemas receptores en términos de toxicidad tanto aguda como crónica, representando una herramienta de gran utilidad en la jerarquización de los efluentes con la consecuente adopción de estrategias y en toma de decisiones en acciones de control (Gherardi-Goldstein et al., 1990). La carga tóxica se estima multiplicando la toxicidad del efluente por su caudal. Los valores de toxicidad son transformados a Unidades Tóxicas (UT) utilizando el índice $\mathrm{EC}_{50}$ o NOEC, de manera de contar con una estimación relacionadas con la toxicidad aguda (UTa) o crónica (UTc).

La carga tóxica aportada por este efluente se estima mediante:

$$
\text { Carga tóxica }=\text { UT } x \text { Qe }
$$

donde $\mathrm{Qe}=$ caudal del efluente, estimado en $300 \mathrm{~m}^{3} /$ hora.

Por lo tanto la carga tóxica asociada a este efluente considerando los efectos agudos y crónicos producidos sobre las especies estudiadas sería:

- Scenedesmus quadricauda

Carga tóxica efectos agudos $=3189$

Carga tóxica efectos crónicos $=19980$

- Raphidocelis subcapitata

Carga tóxica efectos agudos $=2652$

Carga tóxica efectos crónicos $=9675$
Como puede notarse la carga tóxica estimada de este efluente (considerando tanto la producción de efectos agudos como crónicos sobre ambas especies) fue muy importante, ya que un efluente con una toxicidad del $55 \%$ tendría una carga tóxica de 545. Es posible también, estimar el impacto que ocasiona la descarga del efluente en el ambiente receptor. Uno de los métodos propuestos, contempla no solamente la toxicidad del mismo sino también el caudal de descarga del efluente y el caudal mínimo del cuerpo de agua receptor (Walsh \& Merrill, 1984; Gherardi-Goldstein et al., 1990; Grothe et al., 1996). Los mencionados autores recomiendan trabajar con una batería de especies, y seleccionar aquella más sensible a fin de confrontarla con la dilución final del efluente en el ambiente acuático. En este caso estiman, partiendo de la base de que ocurre una mezcla completa inmediatamente después de la descarga de la pluma del efluente, el parámetro denominado Concentración del efluente en el río o CER para el cuerpo de agua receptor, como:

$$
\mathrm{CER}=\left(\mathrm{Qe} /\left(\mathrm{Qe}+\mathrm{Q}_{\mathrm{mR}}\right) \times 100\right.
$$

donde Qe es el caudal del efluente en $\mathrm{m}^{3} / \mathrm{h}$ y $\mathrm{Q}_{\mathrm{mR}}$ el caudal mínimo del río.

Este último valor puede encontrarse en las estadísticas hidrológicas del ambiente en cuestión. En Estados Unidos se utiliza el denominado $\mathrm{Q}_{7,10}$, que es el caudal promedio mínimo a lo largo de 7 días con probabilidad de repetición de 10 años. Recientemente se propuso utilizar el $\mathrm{Q}_{7,2}$, ya que el anterior parámetro se alejaba de los valores actuales (Grothe et al.,1986). Para el caso de la curtiembre, y partiendo de la base de que las algas fuera los organismos más sensibles en una serie de ensayos realizados con, por lo menos 3 especies representativas de los principales niveles tróficos de los ambientes acuáticos, es posible calcular el CER. El valor de Qe fue para el período de toma de muestras de $300 \mathrm{~m}^{3 / h}$ y el caudal del río, extrayendo de los valores presentados por Alberdi et al (1992) un valor mínimo aproximado, durante el período 1993 - 1994, $\mathrm{Q}_{\mathrm{mR}}$ 
igual a $24300 \mathrm{~m}^{3} / \mathrm{h}$. Obtenemos así, una dilución final del efluente en el río de $1.22 \%$.

Según esta estimación el efluente no ejercería efectos sobre el crecimiento de las poblaciones algales aisladas del sistema acuático receptor, luego de 96 horas de exposición, ya que su concentración estimada en el río sería menor a los índices de toxicidad obtenidos para Scenedesmus quadricauda y Raphidocelis subcapitata tanto para los estimadores de la toxicidad agua $\left(\mathrm{EC}_{50}\right)$ como crónica (NOEC, LOEC).

El modelo presentado para estimar la dilución del efluente en el río asume que este es totalmente soluble y que rápidamente se mezcla de modo completo en las proximidades de la descarga y un flujo unidireccional de la corriente de agua (Dorn \& Compernolle, 1995). No tiene en cuenta cuestiones relacionadas con la turbulencia del agua o con la pendiente del terreno por el cual drenan los ríos o inclusive la salinidad y temperatura del efluente que ejercen influencia sobre su densidad, determinando la profundidad de la pluma del mismo.

En virtud de la estimación del CER y las condiciones particulares del río teniendo en cuenta las consideraciones anteriores, se realizaron evaluaciones de toxicidad con muestras líquidas provenientes del río Luján, con el fin de estudiar el impacto que este efluente ejercería sobre este ambiente acuático receptor.

Se recolectaron muestras del río Luján en estaciones ubicadas 10 metros antes y 10 metros después de la descarga del efluente. Los parámetros físico-químicos correspondientes a cada estación indicaron variaciones en la conductividad entre 3300 y $3200 \mu \mathrm{mhos} / \mathrm{cm}$, mientras que no existieron diferencias entre el $\mathrm{pH}$ y la salinidad de las mismas.

Se realizaron evaluaciones de 96 horas de duración utilizando las especies Scenedesmus quadricauda y Raphidocelis subcapitata.

De los resultados obtenidos se pudo comprobar que no existieron diferencias significativas en las densidades celulares de ambas especies al final del período de incubación, expuestas a las muestras tomadas antes y después del vertido del efluente, respecto de los controles. Estos resulta- dos podrían indicar un efecto de dilución por parte de las aguas del río originando una disminución en la salinidad y la conductividad del efluente, con la consecuente reducción en toxicidad. Los parámetros físico-químicos verifican esta situación, ya que a pesar de la elevada conductividad y salinidad del efluente puro $(10500 \mu \mathrm{mhos} / \mathrm{cm} ; 6$ $\%$, respectivamente) no existieron diferencias entre estos parámetros correspondientes a las muestras de agua del río recolectadas antes y después del vuelco del efluente.

\section{CONCLUSIONES}

Los resultados obtenidos permiten concluir que el efluente proveniente de la industria de producción de plásticos y formulación de herbicidas, no produciría efectos tóxicos en las poblaciones algales estudiadas. Este efluente podría ocasionar, en cambio, un aumento de la biomasa algal debido a la presencia de concentraciones elevadas de nitratos y fosfatos. Estas características del efluente final favorecerían el aumento del desarrollo de las poblaciones algales pudiendo aparecer, problemas de eutrofización en el ambiente acuático receptor. Si bien el efluente no resultó tóxico, cabría de esperar efectos no deseados en los ecosistemas receptores. El grado de impacto de este efluente dependerá del grado de trofismo del sistema acuático receptor.

Los resultados obtenidos nos permiten evaluar también el grado de eficiencia del tratamiento de los efluentes respectivos. Las evaluaciones realizadas nos indican que el sistema de depuración de sus efluentes funciona correctamente, ya que el mismo no presenta fitotoxicidad, a pesar de que la formulación de herbicida es una de sus principales actividades. Este buen funcionamiento también se refleja en los valores de los parámetros físico-químicos del efluente final, especialmente la DBO y DQO (Tabla 1). Sin embargo, podríamos afirmar que, en términos ambientales, dicha planta depuradora debería realizar un tratamiento avanzado ó terciario de sus efluentes con el fin de eliminar el exceso en el contenido de nutrientes. 
El efluente menos tóxico para las especies evaluadas resultó ser el proveniente de una industria de producción de colorantes. El efluente de mayor toxicidad correspondió al proveniente de la industria de curtido de cueros.

Respecto a la sensibilidad de las especies utilizadas, la cepa de Scenedesmus quadricauda aislada de las nacientes del río Luján, resultó ser la más sensible, tanto para los índices de toxicidad crónica como aguda.

Las evaluaciones ecotoxicológicas realizadas con muestras líquidas provenientes del río Luján, permiten concluir que existiría una reducción de la toxicidad in situ por efectos de dilución, reduciendo el impacto de este efluente sobre el sistema acuático receptor.

\section{BIBLIOGRAFÍA}

ALBERDI, J. L., M.E. SAENZ, W. D. DI MARZIO y M. D. C. TORTORELLI. 1992. Estudio de la calidad de agua del río Luján. Parte I. Resumos do II Congreso Latinoamericano de Ecología, Caxambú, Brasil. Vol 1, 67-68.

AMBLARD, C., P. COUTURE \& G. BOURDIER. 1990. Effects of a pulp and paper mill effluent on the structure and metabolism of periphytic algae in experimental streams. Aquat. Toxicol., 18: 137162.

APHA. 1992. Standard methods for the examination of water and wastewater. American Public Health Association. 560 pp.

ARAUZO, M, M. F. COLMENAREJO, E. MARTIINEZ \& M. G. GARCÍA. 2000. The role of algae in a deep wastewater self regeneration pond. Wat. Res., 34 (14): 3666-3674.

BRUNO, E. \& B. EKLUND. Two new growth inhibition tets with the filamentous algae Ceramium strictum and C. tunuicorne (Rhodophyta). In press.

BURRIDGE, T. R. \& J. BIDWELL. 2002. Review of the potential use of brown algal ecotoxicological assays in monitoring effluent discharge and pollution in southern Australia. Mar. Poll. Bull., 45: 140-147.

CLAESSON, A. 1984. Use of a mixed algal culture to characterize industrial wastewaters. Ecotoxicol. Environ. Safety, 8: 80-96.
COUTURE, P., C. THELLEN, P. A. THOMPSON \& J. C. AUCLAIR. 1987. Structure and function of phytoplanktonic and microbial communities in relation to industrial wastewater discharge: an ecotoxicological approach in a lotic system. Can. J. Fisheries Aquat. Sci., 44: 167-175.

DE PIETRI, D. E. 1999. Los problemas ambientales de la concentración urbano-industrial. Rev. Ing. Sanitaria-AIDIS, 45: 70-79.

DEL GIORGIO, P. A., A. L. VINOCUR, R. LOMBARDO \& H. G. TELL. 1991. Progressive changes in the structure and dynamics of the phytoplankton community along a pollution gradient in a lowland river- a multivariate approach. Hydrobiologia, 224: 129-154.

DORN, P. B. \& R. VAN COMPERNOLLE. 1995. Effluents. In: Fundamentals of aquatic toxicology: effects, environmental fate and risk assessment. Rand, G.R. (ed.): 903-937. Taylor \& Francis, USA.

EKHOLM, P. \& K. KROGERUS. 1998. Bioavaibility of phosphorus in purified municipal wastewater. Wat. Res., 32 (2): 343- 351.

ELORANTA, P \& R. KETTUNEN. 1979. Phytoplankton in a watercourse polluted by a sulfite cellulose factory Ann. Bot. Fennici, 16: 338-350.

WALSH, G. E., M. D. Kenneth \& R. B. Foster. 1982. Algae and crustaceans as indicators of bioactivity of industrial wastes. Water Res., 16: 879-883.

FERRARI, B., N. PAXÉUS, R. LO GIUDICE \& A. POLLIO, J. GARRIC. Ecotoxicological impact of pharmeceuticals found in treated wasters: study of carbamazepine, clofibric acid and diclofenac. Ecotoxicol. Environ. Safety, In press.

GHERARDI-GOLDSTEIN, E., E. BERTOLETTTI, P. A. ZAGATTO, R. P. ALMEIDA ARAUJO e M. L. L. CASTRO RAMOS. 1990. Procedimentos para utilização de testes de toxicidade no controle de efluentes líquidos. Série Manuais. CETESB (Companhia de Tecnologia de Saneamento Ambiental), Governo do Estado de São Paulo, Secretaria do Meio Ambiente. 17 pp.

GREENE, J. C. \& W. E. MILLER. 1988. Use of Selenastrum capricornutum to assess the toxicity potencial of surface and ground water contamination caused by chromium waste. Environ. Toxicol. Chem., 7: 35-39.

GROTHE, D. R., K. L. DICKSON \& D. K. REEDJUDKINS. 1986. Whole effluent toxicity testing: an evaluation of methods and prediction of receiving system impacts. SETAC Special Publication, SETAC Press, Fl 
JOUBERT, G. 1980. A bioassay application for quatitative toxicity measurements, using the green algae Selenastrum capricornutum. Water Res., 14: 1759-1763.

JUNEAU, P. H. SUMITOMO, S. MATSUI, S. ITOH, S. KIM \& R. POPOVIC. 2003. Use of chlorophyll fluorescence of Closterium ehrenbergii and Lemna gibba for toxic effect evaluation of sewage treatment plant effluent and its hydrophobic components. Ecotoxicol. Environ. Safety, 55: 1-8.

KÄLLQVIST, T. \& A. SVENSON. 2003. Assessment of ammonia toxicity in tests with the microalga, Nephroselmis pyriformis, Chloropyta. Water. Res., 37: 477-484.

LEWIS, M. A. 1992. Periphyton photosynthesis as an indicator of effluent toxicity: relationship to effects on animal test species. Aquat. Toxicol., 23: 279-288.

SÁENZ, M. E. 1992. Evaluación del estado del tratamiento de las aguas residuales en la ciudad de Luján. Influencia de las descargas sobre el río adyacente. Monografía para el curso de postgrado "La potabilización de las aguas. Tratamiento de las aguas residuales e industriales". Depto. Postgrado. Facultad de Ciencias Naturales y Museo, Universidad Nacional de La Plata, 9 pp.

SÁENZ, M. E. 2000. Estudios de los efectos de contaminantes sobre poblaciones algales de agua dulce. Tésis Doctoral, Facultad de Ciencias Naturales y Museo, Universidad Nacional de La Plata. 375 pp.

SPONZA, D. T. 2003. Application of toxicity tests into discharges of the pulp-paper industry in Turkey. Ecotoxicol. Environ. Safety, 54: 74- 86.
TARLAN, E., F. B. DILEK \& U. YETIS. 2002. Effectiveness of algae in the treatment of a woodbased pulp and paper industry wastewater. Biores. Tech., 84: 1-5.

THOMAS, J. M., J. R. SKALSKI, J. F. CLINE, M. C. MCSHANE \& J. C. SIMPSON. 1986. Characterization of chemical waste site contamination and determination of its extent using bioassays. Environ. Toxicol. Chem., 5: 487-501

U. S. ENVIRONMENTAL PROTECTION AGENCY (US EPA). 1989. Selenatrum capricornutum growth test. In: Short-term methods for estimating the chronic toxicity of effluents and receiving water to freshwater organisms. EPA/600/4-89/014. U.S. Environmental Monitoring and Support Laboratory Office of Research and Development, Cincinnati, Ohio.

U. S. ENVIRONMENTAL PROTECTION AGENCY (US EPA). 1996. Algal Toxicity Ecological Effect test guideline. Prevention, Pesticides and Toxic Substance OPPTS 850.5400. EPA 712-C96-164.

WALSH, E. G. \& L. H. BAHNER. 1980. Toxicity of textile mill effluents to freshwater and estuarine algae, crustaceans and fishes. Environ. Pollut., 21: 169-179.

WALSH, G. E \& R. G. MERRILL. 1984. Algal bioassays of industrial and energy process effluents. In: Algae as ecological indicators. L. E. Shubert (ed): 329-359. Academic Press, USA

WEST, INC and D. D, GULLEY. 1996. TOXSTAT ${ }^{\circledR}$ V 3.5. Western Ecosystems Technology Inc. WY. 